\title{
Introduction à la « forme-rapport » : caractéristiques et temporalités d'une production de vérité publique
}

Jean-Louis Fournel

\section{(2) OpenEdition \\ Journals}

Édition électronique

URL : http://journals.openedition.org/conflits/2192

DOI : $10.4000 /$ conflits.2192

ISSN : 1777-5345

Éditeur :

CCLS - Centre d'études sur les conflits lilberté et sécurité, L'Harmattan

Édition imprimée

Date de publication : 1 mai 2007

Pagination : $37-49$

ISBN : 978-2-296-03084-8

ISSN : 1157-996X

Référence électronique

Jean-Louis Fournel, « Introduction à la « forme-rapport » : caractéristiques et temporalités d'une production de vérité publique », Cultures \& Conflits [En ligne], 65 I printemps 2007, mis en ligne le 04 janvier 2010, consulté le 30 mars 2021. URL : http://journals.openedition.org/conflits/2192 ; DOI : https://doi.org/10.4000/conflits.2192 


\title{
Introduction à la « forme-rapport » : caractéristiques et temporalités $d^{\prime}$ une production de vérité publique ${ }_{1}$
}

\author{
Jean-Louis FOURNEL
}

Jean-Louis Fournel, ancien élève de l'ENS de Saint-Cloud, est professeur à l'université Paris-8 et membre de l'UMR 5206 Triangle (ENS LSH Lyon). Spécialiste de l'histoire de la rhétorique et de la pensée politique italienne de la Renaissance, traducteur et commentateur (en collaboration avec Jean-Claude Zancarini) de divers textes de Machiavel, Savonarole et Guicciardini, auteur de nombreux travaux sur l'bistoire de la pensée politique républicaine italienne, il a par ailleurs dirigé entre 1997 et 2001 plusieurs programmes de coopération européenne avec les universités de Bosnie-Herzégovine et fut, avec Pierre Bayard, à l'origine du jumelage de son université avec celle de Sarajevo en 1994, pendant le siège de la ville.

A Fahrudin Kreho, de Bratunac et de Sarajevo

La forme-rapport : considérations préliminaires

F ntre 1999 et 2004 2 , différents rapports sur « l'événement-Srebrenica » ont res (en France et aux Pays-Bas tout du moins), des agences internationales (l'ONU) ou des gouvernements (le rapport du gouvernement néerlandais, qui fut confié à un institut de recherche indépendant - le NIOD - ou le rapport du gouvernement de la Republika Srpska, fermement sollicité par le Haut Représentant de la communauté internationale - OHR - en BosnieHerzégovine, et préparé par une commission mixte d'experts - historiens, fonctionnaires, membres d'ONG). Il s'agira donc à chaque fois d'aborder la

1. Cet article est dédié à Fahrudin Kreho, l’ami sarajévien né sur les bords de la Drina qui nous manque et que je n'oublie pas. Une « version longue » de cet article avec un plus grand nombre de notes est disponible sur le site Internet de Culture $\mathcal{E}$ Conflits : www.conflits.org

2 . Les différents rapports que nous prendrons en compte sont ceux de l'ONU (15 novembre 1999), du Parlement français (novembre 2001), du gouvernement néerlandais (confié au NIOD dès 1996 et rendu en avril 2002), de la RS (juin 2004). Voir la liste des sigles et acronymes en fin d'introduction. 
construction d'un discours «public » émanant d'une instance représentative à un titre ou à un autre.

Tout rapport induit à ce titre un ensemble de caractéristiques formelles de procédure, et surtout de contraintes qui s'imposent à tous les acteurs quelle que soit leur formation intellectuelle et politique. Ce système de contraintes n'est évidemment pas sans effet sur la construction d'un sens et sur les modalités d'interprétation de l'événement. Pour aborder ce que l'on nommera ici schématiquement « la forme-rapport », partons de deux remarques. D'un côté, la comparaison, ou la confrontation, entre les différents rapports contribue à étalonner la fiabilité de chacun d'entre eux et, du même coup, à établir une vérité des faits, fût-elle partielle ou hypothétique, que tous ces rapports se fixent comme objectif premier. D'un autre côté, bien que les textes en question nous en disent autant sur ceux qui les ont rédigés que sur ce dont ils parlent, cela ne doit pas conduire à en négliger la portée. Deux raisons au moins y concourent. Tout d'abord, les institutions qui ont commandé ces rapports sont à divers titres des acteurs de l'événement et y ont une responsabilité à déterminer (ONU, contingents nationaux et chefs de la FORPRONU, parties en conflit, etc.). Le jugement sur leur action, y compris quand il est construit de façon « interne » par une institution à laquelle l'un de ces acteurs se rattache, est l'une des composantes nécessaire de l'analyse de l'événement. La seconde, sans doute plus importante, tient à la forme même du rapport et à sa temporalité propre - le temps qu'il faut pour recueillir les données utiles mais aussi le temps qui le sépare de l'événement fondateur. Cette temporalité peut introduire une faille entre les raisons qui ont conduit à commander le rapport et celles qui vont conduire à l'achever puis à le rendre public, comme si l'institution ne contrôlait pas tout à fait ce qu'elle met en branle, comme si le carcan institutionnel ne pouvait complètement encadrer l'histoire du passé proche, surtout lorsque l'on traite d'un événement d'une telle gravité et d'un tel poids historique.

Dans cette perspective, partir des données formelles communes à l'ensemble des enquêtes et rapports produits sur Srebrenica afin de développer un questionnement sur ces textes ne relève pas d'un quelconque formalisme, qui serait particulièrement malvenu quand on traite d'un massacre de masse. Ces textes officiels participent en effet d'une démarche commune de production d'un discours public de vérité. D'ailleurs, les rapports de la Mission d'information parlementaire française et du NIOD citent et disent utiliser les autres rapports achevés ou en cours, et tous se réfèrent aux travaux et enquêtes du TPIY. Du même coup, ce type de texte répond à un contrat de lecture relativement déterminé, impliquant un cadre prédéfini. La principale de ses spécificités tient au fait que les rapports sont par définition le produit d'un travail collectif dans lequel tout auteur singulier s'efface : le seul cas où s'exprime un « je » est celui du rapport de l'ONU et celui-ci est une première personne du singulier artificielle, dans la mesure où ce n'est évidemment pas le secrétaire général de l'ONU qui a rédigé ledit rapport mais, dans les faits, là encore, un auteur anonyme. 
Il est aisé et nécessaire d'étudier la mise en œuvre de ce contrat de lecture particulier, notamment au travers des écarts qu'il peut présenter au regard de la norme attendue, notamment en terme d'objectivité et de neutralité dans le discours. Ainsi, par exemple, la fonction, la formation intellectuelle, voire l'origine nationale des différentes personnes chargées de l'élaboration des rapports ainsi que les différents moments historiques dans lesquels s'inscrivent leurs pratiques de rédaction peuvent conditionner leurs conclusions respectives. Des questionnements relevant d'une classique analyse du discours sont du même coup susceptibles de nous aider dans l'analyse. Qui rassemble les données et qui rédige le rapport (des parlementaires, des historiens, des policiers, des hauts fonctionnaires internationaux, des hommes de loi) ? Quelle différence introduit chez les rédacteurs la nature variable et évolutive des lecteurs potentiels (tout ou partie d'une communauté nationale, la communauté internationale, les professionnels de la justice, etc.) ? Quelle est la nature des éléments pris en compte dans la démarche gnoséologique (quels types d'indice, de trace, de preuve sont convoqués dans le travail)?

Ultime prémisse, on pourrait croire a priori que l'objet rapport relève d'une certaine "neutralité », qu'il assume une posture « objective », garantie par la distance prise par rapport à l'événement et par la dissociation des fonctions d'acteurs de l'événement et de rédacteur d'une synthèse sur celui-ci. Or, même si cette prétention à l'objectivité est défendue, notamment par les auteurs du rapport du NIOD, les choses s'avèrent plus complexes. D'une part, les rédacteurs ou enquêteurs peuvent à l'occasion être considérés quasiment comme des « acteurs » dès lors qu'ils sont marqués par une expérience personnelle sur le terrain ou qu'ils inscrivent leur activité dans un processus de type judiciaire. C'est le cas de David Harland, fonctionnaire de l'ONU et auteur principal du rapport voulu rapidement par l'organisation internationale sur les « zones de sécurité » (et pas seulement sur Srebrenica), qui a passé la guerre à Sarajevo et a été présent aux négociations qui ont accompagné la chute de Zepa. C'est d'ailleurs sur le rapport de David Harland que se fonde le passage du NIOD consacré à Zepa. On pourrait en dire autant de Jean-René Ruez qui, arrivant sur la scène du crime quelques semaines après le massacre, devient par là même un acteur de l'événement. D'autre part, presque tous les rapports sont conscients des limites de leur travail et des effets négatifs induits par les différentes contraintes temporelles inhérentes à de tels processus, contraintes sur lesquelles nous reviendrons plus loin. La «formerapport » s'affirme ainsi comme un objet hybride et complexe que nous tenterons de cerner en distinguant les modalités ou procédures à l'œuvre, les logiques dépendant des institutions ou des personnes et les problèmes de temporalité.

\section{Modalités et procédure (auditions et enquêtes)}

\section{La double injonction}

Que le rapport se veuille «complet» (ONU), «méthodique» (rapport parlementaire français), ou «systématique» (NIOD), «scientifique» et 
« détaillé » (RS), il s'assigne deux objectifs : apporter une contribution à la "vérité » (le terme revient explicitement dans tous les textes) et évaluer des «responsabilités» morales, politiques ou criminelles éventuelles dans le massacre. La Mission d'information parlementaire du Parlement français entend ainsi développer une "enquête méthodique "s'assignant un double objectif : comprendre la chute de Srebrenica malgré son statut de "zone de sécurité », et étudier le «rôle de la France dans cette tragédie » au regard de l'accusation présentée comme "paradoxale » d'une responsabilité française dans le massacre de masse. Même si Harland considérait que son travail consistait moins à assigner des responsabilités qu'à reconstruire chronologiquement l'ensemble de l'activité de la FORPRONU, le rapport de l'ONU aide en définitive à comprendre quelle est la part de responsabilité des Nations unies dans la chute d'une des «zones de sécurité ». Bien que l'essentiel des questions de responsabilités précises soit confiné dans les parties confidentielles de ce rapport, le rapport de la RS doit dire aux familles des disparus ce que sont devenus leurs proches et, à cet égard, il est conduit à reprendre à son compte une comptabilité significative des victimes ${ }^{3}$. Enfin, le rapport du NIOD et celui du Parlement français entendent analyser l'attitude du «bataillon hollandais » à Srebrenica ou celle du commandement français de la FORPRONU et leurs degrés respectifs de responsabilité dans la chute de l'enclave.

Cette double injonction déclinée dans l'ensemble des rapports et le mélange de ces deux types d'objectifs sont constitutifs d'une ambivalence aussi manifeste que problématique : il s'agit en effet d'emblée de s'adresser à deux destinataires différents (l'un plus universel et «externe », l'autre plus national ou plus « interne»), de mêler à chaque fois des démarches de natures différentes (historique, juridique, morale, administrative, policière) qui ne se recouvrent pas nécessairement et peuvent s'avérer contradictoires. En outre, on peut avoir l'impression (c'est patent dans le cas du rapport du NIOD, et ce n'est pas sans effet dans ceux de l'ONU, de la RS ou du Parlement français) que l'objectif national ou particulier parasite l'autre. Dans une des versions les plus caricaturales de ce conflit d'intérêts, l'épilogue du rapport du NIOD semble accorder plus d'importance aux atteintes à l'image des Pays-Bas à l'étranger qu'à l'histoire du massacre de masse (quelles que puissent être les qualités de différentes parties de ce très long rapport).

\section{La mécanique des auditions}

Pour tous les rapports, le travail s'appuie sur un nombre circonscrit d'auditions, mais avec des procédures différentes de mise en œuvre du travail. Pierre Brana souligne d'ailleurs clairement dans son article que le rapport doit être analysé comme une pièce essentielle et autonome, mais aussi comme l'un

3. Voir l'article de M. Picard et A. Zinbo, ainsi que celui d'I. Delpla dans ce numéro. 
des éléments d'un dispositif plus complexe fondé sur l'articulation d'un « rapport » (moins de 200 pages dans le cas français) avec les procès-verbaux des auditions (600 pages dans ce même cas). C'est ce qui fait que les choses peuvent être différentes quantitativement et qualitativement d'un rapport à l'autre, tant à cause de la taille du rapport qu'à cause de la publicité ou de la confidentialité des auditions. De fait, si le rapport de l'ONU est à peu près de la même ampleur que le rapport français, le rapport du NIOD prend une dimension quasi infinie (près de 7000 pages avec les annexes !). A l'inverse, la version rendue publique du rapport de la RS est très courte (une quarantaine de pages) même si, par ailleurs, ce rapport comprend aussi des annexes confidentielles (et qui peuvent relever d'une logique plus judiciaire).

La tension synthétique du rapport s'oppose ainsi à la nature potentiellement infinie des auditions - leur nombre peut être illimité - et à leur caractère indéfini leur contenu peut prendre toutes les directions possibles. Elles nourrissent ainsi la synthèse mais, en même temps, ne laissent pas de susciter de légitimes interrogations sur le choix nécessairement sélectif des auditionné(e)s et sur le travail également indispensable de réécriture des procès verbaux, si, tout du moins, l'accès aux auditions est rendu possible... En effet, dans le cas des rapports de l'ONU, du NIOD ou de la RS les auditions sont évoquées, la liste des personnes interrogées est même fournie, mais le contenu des entretiens n'est pas rendu public.

Le cas de la Mission d'information parlementaire française est le seul où les auditions sont presque toutes rendues publiques et mises en ligne. C'est aussi le seul où, qui plus est, elles ne sont pas soumises à la confidentialité, du moins lorsqu'elles se passent à Paris et non sur le terrain : sauf demande explicite et fondée de la personne auditionnée, les personnes sont en effet reçues en public par les parlementaires et le cheminement de la réflexion se fait ainsi pour partie en direct et sans médiation ni contrôle. Ainsi, la Mission d'information du Parlement français fonctionne avec une volonté de transparence indéniable. Dans ce seul cas, les auditions échappent donc à un pur statut de pièce subordonnée : l'accès aisé aux procès verbaux des auditions permet d'ailleurs, voire impose, une analyse contrastive et un questionnement sur l'articulation de la publicité et du secret. Encore une fois, les logiques des différents rapports qui sont apparemment similaires peuvent s'avérer assez radicalement divergentes, ce qui n'est d'ailleurs pas sans effets sur leurs conclusions respectives.

\section{Composition et travail des commissions}

Une des raisons pour lesquelles s'instaurent de telles différences tient à la composition des commissions. Dans le cas français, les parlementaires, en tant que représentants du peuple, sont ainsi - et ce très légitimement - à la fois des militants, des élus, des « hommes de la rue » et des responsables politiques. La pluralité des identités, à l'œuvre chez les membres de la Mission d'information parlementaire française, ou la définition plus circonscrite caractérisant les rap- 
ports de l'ONU, de la RS ou du NIOD, sont particulièrement perceptibles dans le choix des auditionné(e)s et dans la nature des questions posées. Le choix des auditionné(e)s dans la plupart de ces rapports - le cas du rapport français sur lequel nous nous sommes davantage attardés étant, à cet égard, le plus manifeste - est souvent évident et bien peu problématisé : sont convoqués ou écoutés ceux qui ont eu des responsabilités officielles (ambassadeurs, généraux, envoyés spéciaux de la presse internationale, responsables d'ONG, etc.), les représentants des victimes (femmes de Srebrenica) ou ceux qui ont fait parler d'eux dans le débat public sur la guerre de Bosnie (opinion makers ou grands témoins). Pour le rapport français, à l'exception d'un cas (Thierry Tardy qui venait de soutenir une thèse sur la FORPRONU), les chercheurs sur les Balkans ou sur la guerre sont ainsi très absents de la liste des auditions (ce qui n'était d'ailleurs pas le cas pour la mission française similaire sur le génocide rwandais).

Le rapport du NIOD constitue un cas à part car il est fondé sur des entretiens quasi systématiques avec les membres du bataillon hollandais de casques bleus, sur de nombreux entretiens avec les victimes ainsi que sur l'avis d'experts. En revanche, pour les autres rapports, la tendance lourde semble encore de privilégier les entretiens avec ceux qui ont eu des responsabilités dans l'histoire de ce moment particulier aux dépens des analystes, voire des témoignages de victimes ou de simples soldats. La construction collective d'une vérité publique, d'une vérité "officielle», sans nuance péjorative, assortie d'une recherche de responsabilités historiques qui ne relèvent pas simplement de l'ordre juridique, conduirait dans cette perspective à mettre l'accent sur les acteurs principaux de l'histoire, de façon presque «spontanée », et sans doute aussi pour des raison de temps et de facilité.

Quant à la nature des questions posées, dans le cas du rapport du Parlement français (qui est le seul où nous pouvons nous prononcer sur ce point, dans la mesure où les procès verbaux d'auditions sont disponibles), si les questions sont parfois très précises et spécifiques, il n'est pas rare qu'elles relèvent d'un degré de généralité ou qu'elles s'appuient sur un fondement moral immédiat ayant donc peu à voir avec une quelconque analyse. Du même coup, il est malaisé de faire apparaître dans le propos des parlementaires la mise au point d'une hiérarchie des causalités. Il en ressort une dialectique parfois surprenante, où l'évolution des questions et des questionnants est à l'occasion d'une simplicité qui confine parfois à la (fausse ?) naïveté, mais qui n'est pas non plus dénuée d'ellipses et de sauts de raisonnements. Ainsi, dans le rapport parlementaire français, les points de vue sur l'attitude du général Janvier et les responsabilités de la chaîne de commandement de l'ONU restent vagues, alors même qu'en différents moments des auditions, voire du rapport, une conclusion semblait se dessiner. Certaines de ces auditions n'ont par ailleurs aucun intérêt pour quiconque possède l'information de tout lecteur assidu d'un quotidien sérieux. 
Mais là encore les choses ne sont pas univoques : cette procédure peut parfois étonner mais elle contribue sans doute à instaurer une certaine liberté de ton, permettant de pointer certaines « erreurs » (y compris à propos d'acteurs presque considérés comme des « icônes » publiques, tel le général Morillon) et à mettre en valeur des questions essentielles, parfois délaissées par des interrogatoires plus sophistiqués, telle la violence faite aux civils. Le registre du rapport français reste bien sûr policé (puisqu'il faut tenter de réunir un consensus dans la commission, fût-ce au prix d'un compromis), mais cela n'interdit pas les opinions tranchées et l'affirmation des dissensions quand un point essentiel est en cause. Pierre Brana nous rappelle que ce sera le cas en définitive - pour Madame Aubert et pour lui-même - sur la question d'un accord éventuel entre le général Janvier, commandant en chef des troupes internationales, et le général Mladic - chef d'état-major et commandant de l'armée de la République serbe auto-proclamée. De même, le seul Pierre Brana se démarque de ses collègues sur le point de l'utilisation du terme de « génocide ", qu'il revendique puisqu'il figure dans le jugement Krstic, en 2001, dont la commission a pu avoir connaissance. L'absence de véritables « recommandations » finales dans le rapport français est aussi une trace de ces positions divergentes : la mission a pu se mettre globalement d'accord sur l'essentiel d'un constat pour informer au mieux la représentation nationale; toutefois, elle n'a pu s'entendre sur une traduction politique de cette information. Soit on y verra une insuffisance du processus, soit on y reconnaitra la nécessaire expression de dissensions démocratiques utiles pour la manifestation ultérieure de la vérité, en d'autres lieux et par d'autres instances.

\section{Logiques des institutions, logiques des personnes}

Le statut de ce type de rapport est aussi à considérer au regard du fonctionnement réglementaire et réglementé des institutions qui les commandent (parlement, gouvernement, organisation internationale, OHR, tribunal) et des mandataires qu'elles se choisissent (commissions ad hoc, centres de recherche universitaire, fonctionnaires spécialisés). Ainsi, dans le cas français, les règles techniques de la logique parlementaire (distinction entre commission d'enquête et mission d'information sur laquelle revient longuement Pierre Brana dans son article) ont certes leur importance, mais elles ne l'emportent pas sur la question des équilibres ou déséquilibres traditionnels dans les relations entre pouvoir exécutif et pouvoir législatif. On comprend alors pourquoi Pierre Brana insiste autant sur les faiblesses endémiques de la culture d'investigation dans les affaires internationales au sein du Parlement français. Les différentes missions d'information, et autres commissions d'enquête, pourraient bien d'ailleurs jouer un rôle important dans le développement d'une culture d'investigation dans un domaine dont on a trop longtemps considéré, notamment en France, qu'il relevait des prérogatives régaliennes du chef de l'Etat ou du gouvernement. Du même coup, pourrait s'instaurer entre l'opinion publique et le pouvoir exécutif, entre l'espace de la publicité absolue et celui du 
secret d'Etat, un autre lieu où les élus jouent vraiment leur rôle de "représentants » du peuple. Ces élus assument la charge de la construction d'une culture d'investigation, à l'origine d'une vérité certes officielle mais qui ne soit pas aux antipodes des convictions s'élaborant de façon autonome dans la sphère de l'opinion publique. Les personnes qui sont membres de la commission deviennent ainsi une variable d'importance.

Dans le rapport hollandais du NIOD, les chercheurs (historiens, sociologues, anthropologues, etc.) déploient une expertise de nature plus abstraite, fondée sur une démarche de «spécialistes » de l'écriture de l'histoire agissant en tant que tels, à la recherche systématique de tous les détails, selon une méthodologie universitaire, la seule contrainte posée à l'élaboration étant le secret imposé à ces chercheurs durant leurs travaux. Dans le rapport de l'ONU, un petit nombre de hauts fonctionnaires internationaux, professionnels de la médiation et du conflit, mettent leur expérience ou leur connaissance de cette catégorie de conflits au service d'une synthèse que le secrétaire général de l'organisation devra faire sienne. Dans le cas de la RS, les membres de la commission sont des experts ${ }^{4}$ ou des politiques acceptés par l'OHR et qui répondent à des injonctions précises (passant à l'occasion par la destitution de hauts responsables de la RS) et fortement explicitées (y compris par l'intermédiaire de communiqués de presse de l'OHR !). Face à ces différents cas de figure, la grande spécificité de la Mission d'information du parlement français tient sans doute, encore une fois, à ce que ses membres sont des élus. Les conséquences de cette situation ne touchent pas seulement la publicité des débats déjà évoquée. Le travail des parlementaires est marqué par la revendication explicite d'une absence de culture et de formation spécialisées sur le sujet considéré et par la volonté de ne pas procéder en spécialistes, mais d'agir avec une forme de virginité méthodologique et historique. C'est là une autre des conséquences de l'identité multiple (et, subsidiairement, du degré d'autonomie individuelle) d'un parlementaire.

Par ailleurs, les modalités de la rédaction du rapport parlementaire français, mais aussi celles des autres rapports, ne peuvent être comprises sans évoquer le lien avec les institutions internationales (ONU, OTAN, TPIY, ONG présentes en Bosnie) ou avec les autres gouvernements (Royaume-Uni, Pays-Bas, Bosnie-Herzégovine) qui sont potentiellement parties prenantes de la démarche, notamment pour ce qui est du recueil des informations. L'intersection délicate de ces différentes sphères (parlements, Etats et gouvernements, institutions internationales, tribunaux) conduit souvent, dans les faits, à des recueils de données établis sur des bases encore largement partielles et non systématiques et met en jeu des temporalités qui peuvent être très différentes.

4. Sur la composition de la commission appelée à préparer le rapport de la RS, on se reportera à l'article de M. Picard et A. Zinbo dans ce numéro. 


\section{Temporalités}

Le frottement des diverses temporalités induites par la forme-rapport est une des constantes de l'ensemble de ce dispositif consistant à rassembler informations, explications ou preuves, tout en entretenant des rapports ambigus avec le problème du jugement. Nous parlons de «frottement » car il ne s'agit ici ni d'une articulation évidente, ni d'un croisement volontaire, ni d'une stratification organisée. Pour la clarté de l'exposé nous nous limiterons à trois des temporalités perceptibles : le temps des événements ; le temps mobilisé dans le rapport (le temps choisi en quelque sorte comme cadre de travail, comme bornes chronologiques du questionnement); enfin, le temps de l'enquêteur.

La première de ces temporalités est manifeste bien que la construction de l'événement soit toujours complexe : c'est celle du massacre, de ces quelques jours de juillet 1995. C'est la temporalité à laquelle les enquêtes du TPIY et de la RS doivent se limiter pour des raisons partiellement identiques. En effet, toutes deux sont tenues explicitement à cette réduction de leur chronologie car leur horizon relève de la recherche des responsabilités criminelles. Elles agissent effectivement sous la pression des réquisits d'une logique judiciaire, redoublée pour la RS par l'autorité du OHR. En revanche, les autres rapports ne veulent pas se limiter à cette temporalité-là dans la mesure même où les questions qu'ils entendent se poser concernent les responsabilités morales et politiques plus que les responsabilités criminelles. Dans cette perspective-là, il existe une interdépendance entre objet du rapport et choix de la temporalité selon que l'on privilégie tels ou tels " acteurs », selon que la primauté va à la responsabilité criminelle ou à la responsabilité politique.

La deuxième temporalité est d'une grande importance, surtout dans cette partie des Balkans où - sans doute encore plus qu'ailleurs -l'Histoire est une inépuisable source d'argumentaires plus ou moins frelatés : choisir de ne traiter que de la guerre en ex-Yougoslavie et de l'explosion du pays a un sens précis. C'est d'ailleurs le choix dominant de ces rapports, un choix entraîné par le rapport de l'ONU. De la même façon, on peut dire que la factualité des exécutions s'inscrit dans une logique qui est donnée par le TPIY. Le rapport de l'ONU semble servir peu ou prou de modèle chronologique à tous les autres, parce qu'il est le premier, mais aussi parce qu'il impose une perspective « internationale », liée à la question des «zones de sécurité » et aux engagements auxquels peuvent se sentir tenus les différents Etats qui sont parties prenantes à la mission de l'ONU en Bosnie. La temporalité explicative se calque sur l'intervention de l'ONU comme acteur de la guerre en Bosnie-Herzégovine. Au contraire, on peut symétriquement insister davantage sur l'héritage titiste, sur celui du dernier conflit mondial, voire sur les guerres du XIX ${ }^{\mathrm{e}}$ siècle et du début $\mathrm{du} \mathrm{XX}^{\mathrm{e}}$ siècle. Ceci n'est évidemment pas sans effet sur la hiérarchisation des causalités que l'on met en place. A cet égard, ce n'est pas pour rien que l'OHR enjoint explicitement la commission préparant le rapport de la RS de ne pas 
remonter au-delà du 10 juillet 1995 dans son travail (probablement échaudé qu'il est par un premier rapport de la RS, réalisé en 2002 et destiné au TPIY, et qui s'attardait beaucoup sur la Seconde Guerre mondiale pour fonder une attitude clairement négationniste à propos des massacres de Srebrenica). Les parlementaires français choisissent de se limiter à une période courte de référence (deux ans environ, de la mise en place des « zones de sécurité », en mars 1993, à juillet 1995), alors que les historiens du NIOD, tout en privilégiant eux aussi une chronologie identique (celle de l'intervention du «bataillon hollandais ", encore plus que celle de l'instauration des zones de sécurité), remontent même ponctuellement jusqu'au XIXe siècle (dans l'annexe au rapport écrite par Ger Duizjings) dans un jeu d'échelle chronologique qui tente d'éclairer partiellement l'événement suivant une logique de « longue durée ».

Mais la temporalité la plus riche d'enseignements pour notre propos est certainement celle de l'enquête et c'est à elle que je voudrais consacrer le dernier volet de cet article. Tous les rapports sont soumis à des contraintes temporelles. Le cas le plus évident est celui du rapport «contraint » par excellence, à savoir celui de la RS : là les requisits temporels sont énumérés explicitement (la commission devait rendre ses conclusions au bout de six mois et procurer des rapports d'étape tous les mois). Mais c'est même le cas pour celui du NIOD qui semblait disposer pourtant d'un temps de travail initialement indéfini. A la fin du processus, il a en effet été soumis à une hâte dommageable dans la rédaction finale: ainsi, afin de répondre à un calendrier gouvernemental néerlandais, l'« épilogue » synthétique rédigé par le responsable de la commission n'a pas été soumis aux autres membres de la commission ni encore moins avalisé par eux. Le temps de la publication - au sens de la procédure qui rend publique l'étude accomplie - peut ainsi presser le temps de l'enquête et de sa formalisation. C'est aussi patent pour les rapports de l'ONU, du Parlement français, de la RS ou pour les enquêtes du TPIY, dont les mandants souhaitent fortement qu'ils soient achevés dans un délai raisonnable. Tout se passe comme si les rapports et les enquêtes étaient soumis à une forme d'urgence, à une temporalité très encadrée, plus propre au travail quotidien des médias ou des administrations qu'à celui de la recherche. Les deux principales variables dans cette perspective sont les suivantes : combien de temps s'est-il écoulé entre événement et préparation du rapport, et entre la préparation et la rédaction du rapport ?

La première des variables semble relancer la vieille question des effets de la distanciation propre à l'historien. Cette distanciation est opposable par exemple à l'urgence du travail de la police qui doit agir tout de suite et le plus vite possible, pour que certaines preuves ne disparaissent pas, dans une sorte de perpétuelle course de vitesse avec les «auteurs » du crime (dont certains passages de l'entretien avec le commissaire Ruez dans ce même numéro sont une bonne illustration). Ce qui est une vertu pour l'historien est un handicap pour le policier. Pourtant, une telle distanciation n'est vraiment méthodologiquement revendiquée que dans le seul cas du rapport du NIOD. Et, de fait, les 
événements qui se déroulent entre 1995 et 2005, indépendamment des enquêtes en cours, tendent à interdire de clore le temps de l'événement ${ }^{5}$. La guerre du Kosovo, la chute de Milosevic, les procès ouverts au TPIY (utilisés notamment en IVe partie, chapitre 2 du rapport du NIOD), les dissensions politiques internes dans la Bosnie-Herzégovine de l'après-Dayton, les changements de présidence et de secrétaires d'Etat aux Etats-Unis, l'émergence de l'hyperterrorisme et de la « guerre contre le terrorisme », pour ne citer que quelques variables évidentes, modifient ainsi notablement la perception de l'événement, mais aussi et surtout le sens qui en émerge et les conditions d'acceptabilité du discours tenu sur celui-ci. Ce n'est ainsi pas un hasard si la formalisation du premier rapport important, celui de l'ONU, est contemporaine de la résolution violente du conflit du Kosovo par une intervention armée de l'OTAN, pour partie approuvée par cette même ONU ${ }^{6}$. N'oublions pas non plus que la chute de Milosevic permet une ouverture partielle des archives serbes, ce qui est rappelé dans le rapport du NIOD. Par là même, la prétention à une quelconque distanciation devient assez peu crédible.

La seconde variable (le temps entre préparation et rédaction du rapport) pose un problème d'historien classique, à savoir l'articulation entre le recueil des sources et la narration. Une sorte de mystique du détail peut ainsi étirer indéfiniment ce temps-ci comme dans le rapport du NIOD. Ces chercheurs sont pris dans une logique d'accumulation, pour laquelle ils sont libres de prendre plusieurs années. Ils peuvent parfois nous donner l'impression d'avoir été occupés avant tout à la construction des archives de leur propre discours. Le risque est grand, dès lors, de déboucher sur un refus de toute causalité forte, qui ne se réfère pas au binôme simpliste realpolitik / moralisme générique ${ }^{7}$. Les membres de chaque commission en viennent ainsi à tenter d'inventer et de construire une relation aux diverses temporalités qui leur soit propre et ne relève ni de la mise à distance, ni de l'immédiateté, ni du travail de la preuve, mais qui doit avoir recours, à la fois et en même temps, à ces différentes modalités. A titre d'exemple, on pourrait dire que, pour le NIOD, cette relation relève pour partie au moins de l'auto-légitimation académique et sociale (comment affirmer la nature unique de son expertise, l'exclusivité de cette expertise et la nécessité sociale de cette expertise) alors que, pour l'ONU, elle relève d'une forme de repentance publique (qui peut d'ailleurs être un des éléments d'un processus de relégitimation de l'institution... ), et que, pour le Parlement

5. Rappelons ici les dates de "publication» des différents rapports : ONU (novembre 1999), Parlement français (novembre 2001), NIOD (avril 2002), RS (juin 2004).

6. Le rapport de l'ONU sur Srebrenica a été préparé au cours de l'année 1999. Par ailleurs, il convient de rappeler que l'interprétation de la résolution 1199 du Conseil de sécurité votée le 23 août 1998, résolution sur laquelle s'est fondée l'intervention armée de l'OTAN au Kosovo au printemps 1999, fait encore débat, notamment pour ce qui concerne l'application de l'article 8 de la charte des Nations unies sur le recours à la force (mais ce n'est pas la question qui nous importe ici).

7. On verra sur ce point l'article d'I. Delpla dans ce numéro. 
français, on peut y voir la construction tâtonnante d'un espace de débat démocratique et d'investigation touchant les traditionnelles fonctions régaliennes. Enfin, pour le rapport de la RS, l'enjeu relève de l'articulation entre, d'un côté, la remise en cause de la symétrie entre «belligérants » (défendue durant toute la guerre et des années après Dayton en Serbie et ailleurs... ) et, de l'autre, la recherche d'une forme d'admission du crime de masse qui ne détruise pas la légitimité de la RS, en faisant porter toute la responsabilité sur des individus.

La confrontation entre les effets de cette temporalité spécifique du travail des commissions avec les divers nouds qui restent dans les rapports met en évidence la constante oscillation entre l'information à recueillir (sans en avoir les moyens, le temps, la formation, voire les compétences) et le jugement, explicite ou implicite, à porter (sans en avoir les prérogatives, ni toujours la volonté).

L'attitude des commissions à l'égard de la question du « jugement » est d'ailleurs variable : si les rapports du NIOD et de la RS n'entendent pas s'engager sur cette voie, le rapport de l'ONU et celui du Parlement français, sans parler évidemment des enquêtes du TPIY, revendiquent de travailler pour un jugement, y compris pénal, et qui n'entend pas se limiter à l'hégélien tribunal de l'histoire. "L'engagement global de l'ONU à mettre un terme aux conflits, loin d'exclure les jugements les rend nécessaires » proclame le rapport du secrétaire général dans son avant-dernière phrase. Le rapport parlementaire français a voulu quant à lui "rétablir les faits" parce que les victimes «méritent la vérité non la polémique, la démagogie et le manichéisme " 8 et conclut en «exigeant " que "Français, Britanniques et Américains, notamment, consacrent les moyens nécessaires à la capture de ces criminels contre l'bumanité» 9. Au regard de cela, le rapport du NIOD ne veut être qu'une "monographie analytique» qui ne se situe pas dans "l'arène politique» et «le débat public 10 et renonce à tout «jugement politique».

Maintes ambiguités demeurent donc, qu'elles soient de nature épistémologique (modalités du recueil des données, de leur sélection et de la rédaction; construction d'une légitimité de l'auteur collectif), institutionnelle (degré d'autonomie et d'auto-censure / censure), judiciaire (statut de l'information publiée et utilisation publique des résultats), historique (crédibilité de la reconstitution des causalités) et, enfin, politique (effets induits par le rapport dans les opinions publiques concernées et/ou chez les gouvernants). Ce qui se construit dans les rapports relève d'un objet dont la nature est radicalement hybride et instable (au sens chimique du terme), tout à la fois historique, policière, juridique, politique, pédagogique.

8. Voir p. 151.

9. Voir pp. 151-152.

10. Introduction, p. 10. 
La complexité des temporalités à l'œuvre dans la construction d'un rapport est un facteur essentiel d'explication de cette hybridation et, surtout, de la faiblesse quasiment naturelle du rapport d'information (tout comme de son statut radicalement toujours inachevé). Les rapports encouragent une défense et une illustration de la complexité, ce qui paradoxalement joue à la fois au bénéfice de la recherche d'une vérité des faits et, parfois, aux dépens d'une saisie du sens historique. Les rapports sont pour la manifestation de la vérité, à la fois et indissolublement, indispensables et problématiques, minés qu'ils sont par une certaine défaillance structurelle, c'est-à-dire par les lacunes propres à leur forme, en liaison étroite avec les contraintes qui déterminent leur nature, telles que nous avons tenté de les mettre en évidence plus haut. L'objet rapport étant le produit de contraintes, notamment de réponse à un mandat d'écriture collégiale et de durée imposée à l'enquête et à la publication, des effets manifestes sont créés, antérieurement au positionnement des rédacteurs, quels que soient leur appartenance politique ou leur profil sociologique.

Toute commission peut avoir ainsi tendance, au fil du travail collectif et de la recherche d'un consensus minimal entre les participants, à « raboter » ses analyses, à tel point qu'elle en édulcore le sens profond, voire la pertinence historique. Mais c'est justement la conscience nécessaire de cette faiblesse qui peut rendre indispensables ces rapports. Ne voyons là rien de paradoxal. En effet, seule l'acceptation et l'analyse de cette fragilité, en en tirant toutes les conséquences, permet de faire le départ entre ce qui restera et ce qui devra être écarté de chacun de ces rapports et donc de mettre au jour l'enseignement d'un événement terrible, un enseignement tout ensemble particulier et universel : particulier pour le fait considéré et universel pour les valeurs, valeurs d'éthique et d'analyse, que le fait mobilise à très juste titre. 